\title{
Perioperative outcomes of combined heart surgery and lung tumor resection: a systematic review and meta-analysis
}

\author{
Shizhao Cheng ${ }^{1} \mathbb{D}$, Yiyao Jiang ${ }^{2}$, Xin $\mathrm{Li}^{1}$, Xike Lu${ }^{1}$, Xun Zhang ${ }^{1}$ and Daqiang Sun ${ }^{{ }^{*}}$
}

\begin{abstract}
Objective: The prevalence of patients with concomitant heart and lung lesions requiring surgical intervention is increasing. Simultaneous cardiac surgery and pulmonary resection avoids the need for a second operation. However, there are concerns regarding the potentially increased mortality and complication rates of simultaneous surgery and the adequacy of lung exposure during heart surgery. Therefore, we performed a meta-analysis to evaluate the perioperative mortality and complication rates of combined heart surgery and lung tumor resection.
\end{abstract}

Methods: A comprehensive literature search was performed in July 2020. The PubMed, Embase, and Web of Science databases were searched to identify studies that reported the perioperative outcomes of combined heart surgery and lung tumor resection. Two reviewers independently screened the studies, extracted data, and assessed the risk of bias of included studies. Pooled proportions and $95 \%$ confidence intervals $(95 \% \mathrm{Cl})$ were calculated by R version 3.6.1 using the meta package.

Results: A total of 536 patients from 29 studies were included. Overall, the pooled proportion of operative mortality was 0.01 (95\% Cl: $0.00,0.03$ ) and the pooled proportion of postoperative complications was 0.40 ( $95 \%$ Cl: $0.24,0.57$ ) for patients who underwent combined cardiothoracic surgery. Subgroup analysis by lung pathology revealed that, for patients with lung cancer, the pooled proportion of anatomical lung resection was $0.99(95 \% \mathrm{Cl}: 0.95,1.00)$ and the pooled proportion of systematic lymph node dissection or sampling was $1.00(95 \% \mathrm{Cl}: 1.00,1.00)$. Subgroup analysis by heart surgery procedure found that the pooled proportion of postoperative complications of patients who underwent coronary artery bypass grafting (CABG) patients using the off-pump method was 0.17 ( $95 \% \mathrm{Cl}: 0.01,0.43$ ), while the pooled proportion of complications after CABG using the on-pump method was 0.61 (95\% Cl: 0.38, 0.82).

Conclusion: Combined heart surgery and lung tumor resection had a low mortality rate and an acceptable complication rate. Subgroup analyses revealed that most patients with lung cancer underwent uncompromised anatomical resection and mediastinal lymph node sampling or dissection during combined cardiothoracic surgery, and showed off-pump CABG may reduce the complication rate compared with on-pump CABG. Further researches are still needed to verify these findings.

Keywords: Heart surgery, Lung surgery, Perioperative outcomes, Combined cardiothoracic surgery, Meta-analysis

*Correspondence: sdamd@163.com

${ }^{1}$ Department of Thoracic Surgery, Tianjin Chest Hospital, Tianjin 300222, China

Full list of author information is available at the end of the article

\section{Introduction}

Although relatively few patients have concomitant lesions of the heart and lungs requiring surgical intervention, the prevalence of such patients is increasing [1-3]. Some patients, who required cardiac surgery, found asymptomatic indeterminate lung nodules during original author(s) and the source, provide a link to the Creative Commons licence, and indicate if changes were made. The images or other third party material in this article are included in the article's Creative Commons licence, unless indicated otherwise in a credit line to the material. If material is not included in the article's Creative Commons licence and your intended use is not permitted by statutory regulation or exceeds the permitted use, you will need to obtain permission directly from the copyright holder. To view a copy of this licence, visit http://creativecommons.org/licenses/by/4.0/. The Creative Commons Public Domain Dedication waiver (http://creativecommons.org/publicdomain/zero/1.0/) applies to the data made available in this article, unless otherwise stated in a credit line to the data. 
preoperative examination. Other patients, who were scheduled to receive lung tumor resection, had heart diseases requiring surgical intervention. The management of patients who require both heart surgery and lung resection is challenging. Treatment options include either simultaneous or staged surgical procedures [4-8]. Without postponing the treatment of either heart or lung diseases, simultaneous surgery can solve heart and lung lesions at the same time, thus avoiding the second operation [3, 9-12]. However, there are concerns regarding the potentially increased mortality and complication rates of simultaneous surgery and the adequacy of lung exposure during heart surgery $[8,12-20]$.

To address these concerns, we conducted this metaanalysis to systematically evaluate the perioperative mortality and complication rates of combined heart surgery and lung tumor resection. In the subgroup analyses, we also investigated the proportion of patients with lung cancer who underwent anatomic lung resection and mediastinal lymph node sampling or dissection, and the impact of cardiopulmonary bypass $(\mathrm{CPB})$ on perioperative complications.

\section{Methods}

\section{Search strategy}

A comprehensive literature search was conducted using PubMed, Embase and Web of Science database in 7 July 2020 to identify citations reporting perioperative outcomes of combined heart surgery and lung tumor resection. The following search algorithm was used: (exp Cardiac Surgical Procedures/ or ((heart\$ or cardio\$ or cardiac or coronary or myocardial or valve $\$$ ) adj1 (surger\$ or operation\$ or graft\$ or bypass\$ or revascularization $\$$ or plasty or replacement)).tw.) and (exp Pneumonectomy/ or ( (lung\$ or pulmon\$ or sublob\$ or wedge\$) adj1 (surger\$ or operation\$ or resect\$)).tw.). In addition, the reference lists of the retrieved articles and related reviews were checked to identify potentially relevant additional studies. No language or publication date restrictions were adopted. The flow diagram was presented in Fig. 1. The present meta-analysis was designed, performed, and reported in accordance with the standards of quality for reporting meta-analysis.

\section{Selection criteria}

Articles included in this study had to meet all the following criteria: the exposure of interest was combined heart surgery and lung tumor resection whether benign or malignant; the outcome of interest was the rates of operative mortality and postoperative complications; the study design was a case-control, retrospective, or prospective study; and the proportion estimates with

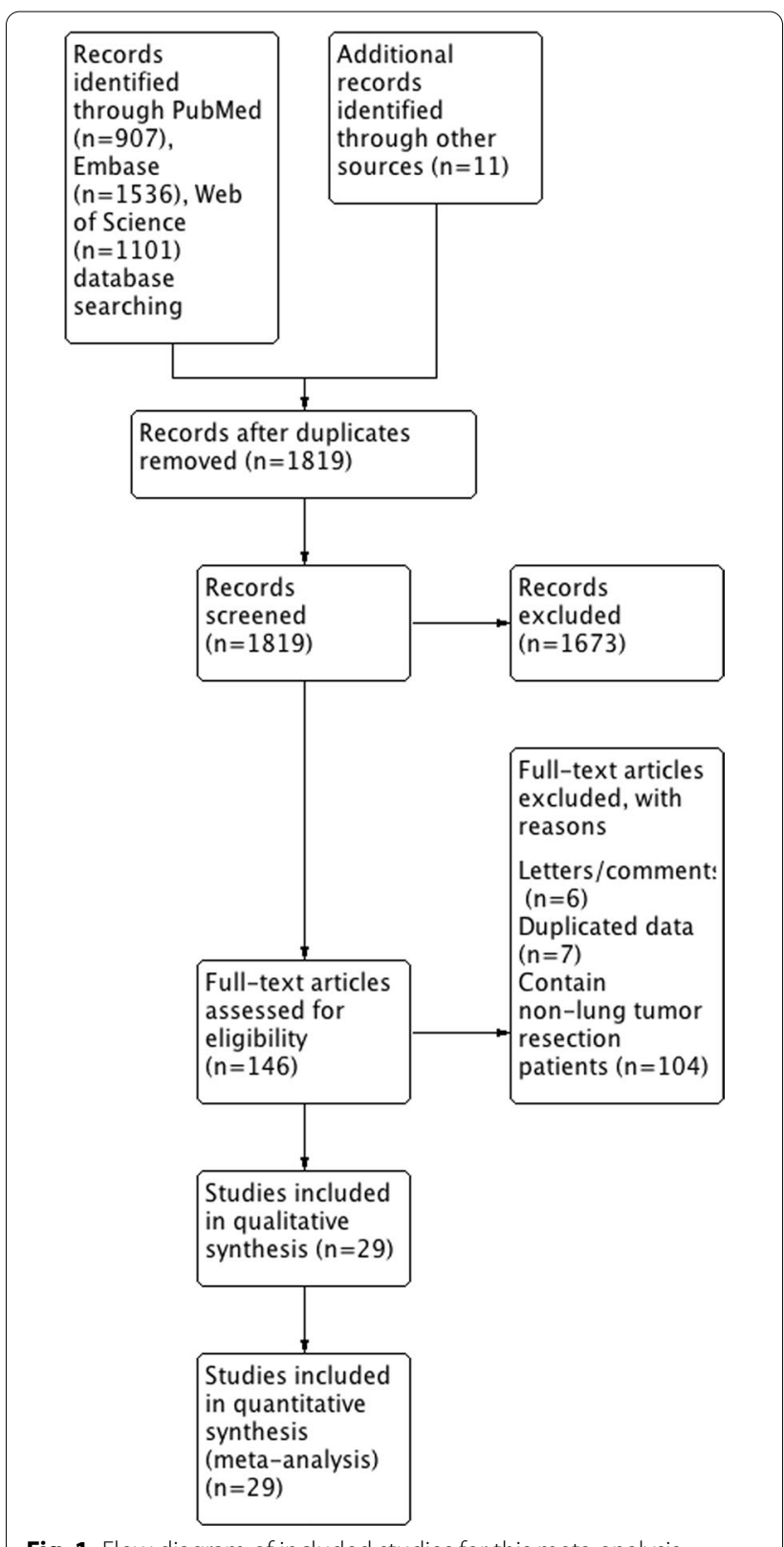

Fig. 1 Flow diagram of included studies for this meta-analysis

their corresponding 95\% confidence intervals (95\% CI) were reported or sufficient data were provided to calculate them. Operative mortality was defined as death within 30 days of operation or during hospitalization. Postoperative complications were defined as the occurrence of complications within 30 days of operation or during the postoperative stay.

The included studies focused on lung tumor resection. Publications were excluded if they involved lung transplantation surgery, trauma surgery, lung infectious disease surgery, esophageal surgery, or mediastinal 
surgery. Reviews, editorial commentaries, or studies with no extractable data were also excluded.

\section{Quality assessment}

Newcastle-Ottawa Scale (NOS) was used to evaluate the quality of included researches by two independent reviewers (Yiyao Jiang, Xin Li). NOS is a 9-star system, which includes 3 dimensions: selection (4 items), comparability ( 1 item), and exposure/outcome (3 items). Each item represents 1 point, except for comparability (2 points). A study with $\geq 7$ points was considered as a highquality study.

\section{Data extraction}

Data were extracted independently by the two reviewers (Shizhao Cheng, Yiyao Jiang) and any discrepancies were resolved by discussion. The following data were extracted from each included study: publication details, sample size, lung tumor histological type, and operative mortality and postoperative complication proportion estimates with their corresponding 95\% CI.

\section{Statistical analysis}

All quantitative analysis was performed in $\mathrm{R}$ version 3.6.1 using the meta package. For the meta-analysis of the operative mortality and postoperative complications proportions, both fixed-effect and random-effect approaches were adopted using the Freeman-Tukey double arcsine transformation method [21]. Subgroup analyses were performed based on different lung pathology or heart surgery procedures. Heterogeneity across included studies was assessed with the Cochran Q test (the level of significance was set at 0.1 ) [22]. The $\mathrm{I}^{2}$ score was used to determine the degree of heterogeneity $\left(\mathrm{I}^{2}<50 \%\right.$, no obvious heterogeneity; $\mathrm{I}^{2}>50 \%$, large or extreme heterogeneity). In sensitivity analysis, meta-analysis was conducted after omitting each study in turn. Potential publication bias was evaluated using Begg's test and Egger's test [23].

\section{Results}

\section{Literature search and study characteristics}

The detailed process of literature retrieval procedures is shown in Fig. 1. A total of 29 eligible studies were finally included in the present meta-analysis [3, 5-20, 24-35]. These studies were carried out in the following geographical regions: five in the USA [8, 19, 20, 32, 35], four in China $[3,11,25,26]$, four in the Netherlands [7, $13,30,33]$, three in Italy $[14,18,29]$, two in the Czech Republic [12, 24], two in Japan [10, 16], two in Turkey $[9,15]$, one in Australia [34], one in Belgium [6], one in Canada [31], one in Germany [5], one in Greece [27], one in Poland [28], and one in the UK [17]. All included studies were retrospective observational studies, which were published between 1990 and 2019. Of these studies, the sample sizes ranged from 2 to 79 patients. Therefore, 29 studies containing 536 patients who received combined heart surgery and lung tumor resection were subjected to final analysis. In each study, the proportion estimates with their corresponding 95\% CIs of the operative mortality and postoperative complications were calculated from available information from original article. Quality scores evaluated by the Newcastle-Ottawa Scale (NOS) ranged from 5 to 7 . The main characteristics of all included studies have been summarized in Table 1 .

\section{Pooled proportion of operative mortality}

The pooled proportion of operative mortality was 0.01 (95\% CI: $0.00,0.03)$ using both fixed-effect and randomeffect models. There was no significant heterogeneity between studies $\left(\mathrm{I}^{2}=0.00 \%, P=0.79\right)$ (Fig. 2).

\section{Pooled proportion of postoperative complications}

The pooled proportion of postoperative complications was 0.40 (95\% CI: $0.24,0.57)$ using the random-effect model. There was significant heterogeneity between studies $\left(\mathrm{I}^{2}=91.60 \%, P<0.01\right)$ (Fig. 3). Furthermore, we divided the postoperative complications into three categories: reopening for bleeding; respiratory complications and cardiac complications. The pooled proportion of reopening for bleeding was 0.01 (95\% CI: 0.00, 0.02) using both fixed-effect and random-effect models, with no significant heterogeneity between studies $\left(\mathrm{I}^{2}=2.10 \%\right.$, $P=0.43$ ) (Fig. 4). The pooled proportion of respiratory complications was 0.11 (95\% CI: 0.04, 0.19) using the random-effect model, with significant heterogeneity between studies $\left(\mathrm{I}^{2}=76.00 \%, P<0.01\right)$. The pooled proportion of cardiac complications was 0.15 (95\% CI: 0.08, $0.23)$ using the random-effect model, with significant heterogeneity between studies $\left(\mathrm{I}^{2}=72.00 \%, P<0.01\right)$.

\section{Subgroup analyses}

To further assess the incidence rates of operative mortality and postoperative complications in patients who received combined heart surgery and lung tumor resection, we classified all patients into subgroups based on lung pathology and heart surgery procedures.

In the subgroups classified by lung pathology, 16 studies [3, 7-9, 11, 13-16, 19, 26, 28, 30, 32-34] contained 327 patients who received combined heart surgery and lung cancer resection, while the other 13 studies $[5,6,10,12$, $17,18,20,24,25,27,29,31,35]$ enrolled patients with both malignant and benign lung diseases. Because malignant and benign lung diseases were analyzed together in these 13 studies $[5,6,10,12,17,18,20,24,25,27,29$, $31,35]$, further assessment of benign diseases was not done. Therefore, we quantitatively pooled the data of 
Table 1 Characteristics of studies included in this meta-analysis

\begin{tabular}{|c|c|c|c|c|c|c|c|c|c|}
\hline Study & Year & Country & $\begin{array}{l}\text { Study } \\
\text { design }\end{array}$ & $\begin{array}{l}\text { Study } \\
\text { period }\end{array}$ & $\begin{array}{l}\text { No. of } \\
\text { patients }\end{array}$ & $\begin{array}{l}\text { Lung pathology } \\
\text { (Adenocarcinoma/ } \\
\text { squamous cell } \\
\text { carcinoma/other } \\
\text { malignant tumors/ } \\
\text { benign tumors) }\end{array}$ & $\begin{array}{l}\text { Heart } \\
\text { surgery } \\
\text { (CABG/ } \\
\text { other types } \\
\text { of heart } \\
\text { surgery) }\end{array}$ & $\begin{array}{l}\text { Operative } \\
\text { mortality } \\
\text { proportions } \\
(95 \% \mathrm{Cl})\end{array}$ & $\begin{array}{l}\text { Postoperative } \\
\text { complication } \\
\text { proportions } \\
(95 \% \mathrm{Cl})\end{array}$ \\
\hline $\mathrm{Li}$ & 2019 & China & Case-control & 2009-2016 & 20 & $13 / 6 / 1 / 0$ & $20 / 0$ & $\begin{array}{l}0.00[0.00 ; \\
0.17]\end{array}$ & $0.00[0.00 ; 0.17]$ \\
\hline Yeginsu & 2018 & Turkey & Retrospective & 2014-2018 & 10 & $3 / 6 / 1 / 0$ & $10 / 0$ & $\begin{array}{l}0.10[0.00 ; \\
0.45]\end{array}$ & $0.40[0.12 ; 0.74]$ \\
\hline Kaku & 2017 & Japan & Retrospective & $2008-2013$ & 18 & $13 / 2 / 0 / 3$ & $6 / 12$ & $\begin{array}{l}0.00[0.00 \\
0.19]\end{array}$ & $0.00[0.00 ; 0.19]$ \\
\hline $\mathrm{Ma}$ & 2016 & China & Retrospective & 2003-2014 & 34 & 18/16/0/0 & $34 / 0$ & $\begin{array}{l}0.00[0.00 ; \\
0.10]\end{array}$ & $0.32[0.17 ; 0.51]$ \\
\hline Santavy & 2015 & $\begin{array}{l}\text { Czech } \\
\text { Republic }\end{array}$ & Retrospective & 2010-2014 & 10 & $3 / 0 / 3 / 4$ & $6 / 4$ & $\begin{array}{l}0.00[0.00 ; \\
0.31]\end{array}$ & $0.10[0.00 ; 0.45]$ \\
\hline Kovacicova & 2014 & $\begin{array}{l}\text { Czech } \\
\text { Republic }\end{array}$ & Case-control & $2002-2011$ & 12 & $1 / 7 / 4 / 0$ & $9 / 3$ & $\begin{array}{c}0.00[0.00 ; \\
0.26]\end{array}$ & $0.75[0.43 ; 0.95]$ \\
\hline Zhang & 2012 & Germany & Retrospective & 1999-2007 & 30 & $6 / 5 / 1 / 18$ & $17 / 13$ & $\begin{array}{l}0.07[0.01 \\
0.22]\end{array}$ & $0.10[0.02 ; 0.27]$ \\
\hline Li & 2012 & China & Retrospective & $2008-2010$ & 3 & $1 / 2 / 0 / 0$ & $3 / 0$ & $\begin{array}{l}0.00[0.00 ; \\
0.71]\end{array}$ & $0.00[0.00 ; 0.71]$ \\
\hline Zhang & 2009 & China & Retrospective & $2003-2008$ & 7 & $2 / 5 / 0 / 0$ & $7 / 0$ & $\begin{array}{l}0.00[0.00 \\
0.41]\end{array}$ & $0.14[0.00 ; 0.58]$ \\
\hline Cathenis & 2009 & Belgium & Retrospective & 2000-2008 & 27 & $10 / 14 / 2 / 1$ & $22 / 5$ & $\begin{array}{l}0.00[0.00 ; \\
0.13]\end{array}$ & $1.00[0.87 ; 1.00]$ \\
\hline Prokakis & 2008 & Greece & Retrospective & 2004-2006 & 5 & $1 / 2 / 0 / 2$ & $1 / 4$ & $\begin{array}{l}0.00[0.00 ; \\
0.52]\end{array}$ & $0.40[0.05 ; 0.85]$ \\
\hline Dyszkiewicz & 2008 & Poland & Retrospective & 2001-2006 & 25 & $6 / 14 / 5 / 0$ & $25 / 0$ & $\begin{array}{l}0.00[0.00 ; \\
0.14]\end{array}$ & $0.64[0.43 ; 0.82]$ \\
\hline $\begin{array}{l}\text { Schoenmak- } \\
\text { ers }\end{array}$ & 2007 & Netherlands & Case-control & 1994-2005 & 43 & $17 / 17 / 9 / 0$ & $37 / 6$ & $\begin{array}{l}0.07[0.01 \\
0.19]\end{array}$ & $0.67[0.51 ; 0.81]$ \\
\hline Caimmi & 2006 & Italy & Retrospective & 2005-2006 & 2 & $1 / 1 / 0 / 0$ & $2 / 0$ & $\begin{array}{l}0.00[0.00 ; \\
0.84]\end{array}$ & $0.00[0.00 ; 0.84]$ \\
\hline Patane & 2002 & Italy & Retrospective & 1991-1999 & 11 & $2 / 7 / 0 / 2$ & $6 / 5$ & $\begin{array}{l}0.00[0.00 ; \\
0.28]\end{array}$ & $0.00[0.00 ; 0.28]$ \\
\hline Koksal & 2002 & Turkey & Retrospective & $2001-2002$ & 2 & 0/2/0/0 & $2 / 0$ & $\begin{array}{l}0.00[0.00 ; \\
0.84]\end{array}$ & $0.00[0.00 ; 0.84]$ \\
\hline Morishita & 2001 & Japan & Retrospective & 1986-2000 & 6 & $3 / 2 / 1 / 0$ & $4 / 2$ & $\begin{array}{l}0.00[0.00 ; \\
0.46]\end{array}$ & $0.83[0.36 ; 1.00]$ \\
\hline Mariani & 2001 & Netherlands & Retrospective & 1999-2000 & 3 & $2 / 1 / 0 / 0$ & $3 / 0$ & $\begin{array}{l}0.00[0.00 ; \\
0.71]\end{array}$ & $0.00[0.00 ; 0.71]$ \\
\hline Danton & 1998 & UK & Retrospective & 1990-1997 & 13 & $6 / 3 / 2 / 2$ & $11 / 2$ & $\begin{array}{l}0.00[0.00 ; \\
0.25]\end{array}$ & $0.62[0.32 ; 0.86]$ \\
\hline Voets & 1997 & Netherlands & Case-control & 1988-1995 & 24 & $8 / 14 / 2 / 0$ & $24 / 0$ & $\begin{array}{l}0.21[0.07 ; \\
0.42]\end{array}$ & $0.79[0.58 ; 0.93]$ \\
\hline Rao & 1996 & Canada & Retrospective & 1982-1995 & 30 & $10 / 5 / 6 / 9$ & $24 / 6$ & $\begin{array}{l}0.07[0.01 ; \\
0.22]\end{array}$ & $0.23[0.10 ; 0.42]$ \\
\hline Francesca & 1995 & USA & Retrospective & 1973-1990 & 21 & $11 / 7 / 3 / 0$ & $18 / 3$ & $\begin{array}{l}0.05[0.00 ; \\
0.24]\end{array}$ & $0.29[0.11 ; 0.52]$ \\
\hline Brutel & 1995 & Netherlands & Retrospective & 1979-1993 & 79 & $22 / 48 / 9 / 0$ & $69 / 10$ & $\begin{array}{l}0.06[0.02 ; \\
0.14]\end{array}$ & $0.86[0.76 ; 0.93]$ \\
\hline Terzi & 1994 & Italy & Retrospective & 1980-1993 & 10 & $2 / 3 / 3 / 2$ & $10 / 0$ & $\begin{array}{l}0.10[0.00 ; \\
0.45]\end{array}$ & $0.40[0.12 ; 0.74]$ \\
\hline Miller & 1994 & USA & Case-control & 1965-1992 & 30 & 19/8/3/0 & $23 / 7$ & $\begin{array}{l}0.07[0.01 ; \\
0.22]\end{array}$ & $0.50[0.31 ; 0.69]$ \\
\hline Yokoyama & 1993 & USA & Retrospective & 1988-1992 & 11 & $6 / 4 / 1 / 0$ & $9 / 2$ & $\begin{array}{l}0.00[0.00 ; \\
0.28]\end{array}$ & $0.64[0.31 ; 0.89]$ \\
\hline
\end{tabular}


Table 1 (continued)

\begin{tabular}{|c|c|c|c|c|c|c|c|c|c|}
\hline Study & Year & Country & $\begin{array}{l}\text { Study } \\
\text { design }\end{array}$ & $\begin{array}{l}\text { Study } \\
\text { period }\end{array}$ & $\begin{array}{l}\text { No. of } \\
\text { patients }\end{array}$ & $\begin{array}{l}\text { Lung pathology } \\
\text { (Adenocarcinoma/ } \\
\text { squamous cell } \\
\text { carcinoma/other } \\
\text { malignant tumors/ } \\
\text { benign tumors) }\end{array}$ & $\begin{array}{l}\text { Heart } \\
\text { surgery } \\
\text { (CABG/ } \\
\text { other types } \\
\text { of heart } \\
\text { surgery) }\end{array}$ & $\begin{array}{l}\text { Operative } \\
\text { mortality } \\
\text { proportions } \\
(95 \% \mathrm{Cl})\end{array}$ & $\begin{array}{l}\text { Postoperative } \\
\text { complication } \\
\text { proportions } \\
(95 \% \mathrm{Cl})\end{array}$ \\
\hline Rosalion & 1993 & Australia & Retrospective & 1987-1990 & 10 & $4 / 5 / 1 / 0$ & $10 / 0$ & $\begin{array}{l}0.00[0.00 ; \\
0.31]\end{array}$ & $0.80[0.44 ; 0.97]$ \\
\hline Ulicny & 1992 & USA & Retrospective & 1980-1990 & 19 & $4 / 0 / 6 / 9$ & $14 / 5$ & $\begin{array}{l}0.05[0.00 ; \\
0.26]\end{array}$ & $1.00[0.82 ; 1.00]$ \\
\hline Canver & 1990 & USA & Retrospective & 1982-1988 & 21 & $4 / 4 / 0 / 13$ & $18 / 3$ & $\begin{array}{c}0.05[0.00 \\
0.24]\end{array}$ & $0.10[0.01 ; 0.30]$ \\
\hline
\end{tabular}

\begin{tabular}{|c|c|c|c|c|c|c|c|c|c|c|}
\hline Study & \multicolumn{2}{|c|}{ Events Total } & & & & & Proportion & $95 \%-\mathrm{Cl}$ & $\begin{array}{r}\text { Weight } \\
\text { (fixed) }\end{array}$ & $\begin{array}{c}\text { Weight } \\
\text { (random) }\end{array}$ \\
\hline Li 2019 & 0 & 20 & 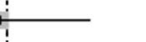 & & & & 0.00 & {$[0.00 ; 0.17]$} & $3.7 \%$ & $3.7 \%$ \\
\hline Yeginsu 2018 & 1 & 10 & 世 & & & & 0.10 & {$[0.00 ; 0.45]$} & $1.9 \%$ & $1.9 \%$ \\
\hline Kaku 2017 & 0 & 18 & & & & & 0.00 & {$[0.00 ; 0.19]$} & $3.4 \%$ & $3.4 \%$ \\
\hline Ma 2016 & 0 & 34 & 5 & & & & 0.00 & {$[0.00 ; 0.10]$} & $6.3 \%$ & $6.3 \%$ \\
\hline Santavy 2015 & 0 & 10 & & & & & 0.00 & {$[0.00 ; 0.31]$} & $1.9 \%$ & $1.9 \%$ \\
\hline Kovacicova 2014 & 0 & 12 & & & & & 0.00 & {$[0.00 ; 0.26]$} & $2.3 \%$ & $2.3 \%$ \\
\hline Zhang 2012 & 2 & 30 & 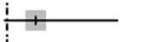 & & & & 0.07 & {$[0.01 ; 0.22]$} & $5.5 \%$ & $5.5 \%$ \\
\hline Li 2012 & 0 & 3 & & & & & 0.00 & {$[0.00 ; 0.71]$} & $0.6 \%$ & $0.6 \%$ \\
\hline Zhang 2009 & 0 & 7 & & & & & 0.00 & {$[0.00 ; 0.41]$} & $1.4 \%$ & $1.4 \%$ \\
\hline Cathenis 2009 & 0 & 27 & is & & & & 0.00 & {$[0.00 ; 0.13]$} & $5.0 \%$ & $5.0 \%$ \\
\hline Prokakis 2008 & 0 & 5 & & & & & 0.00 & {$[0.00 ; 0.52]$} & $1.0 \%$ & $1.0 \%$ \\
\hline Dyszkiewicz 2008 & 0 & 25 & 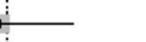 & & & & 0.00 & {$[0.00 ; 0.14]$} & $4.6 \%$ & $4.6 \%$ \\
\hline Schoenmakers 2007 & 3 & 43 & 1 & & & & 0.07 & {$[0.01 ; 0.19]$} & $7.9 \%$ & $7.9 \%$ \\
\hline Caimmi 2006 & 0 & 2 & & & & & 0.00 & {$[0.00 ; 0.84]$} & $0.5 \%$ & $0.5 \%$ \\
\hline Patane 2002 & 0 & 11 & & & & & 0.00 & {$[0.00 ; 0.28]$} & $2.1 \%$ & $2.1 \%$ \\
\hline Koksal 2002 & 0 & 2 & & & & & 0.00 & {$[0.00 ; 0.84]$} & $0.5 \%$ & $0.5 \%$ \\
\hline Morishita 2001 & 0 & 6 & & & & & 0.00 & {$[0.00 ; 0.46]$} & $1.2 \%$ & $1.2 \%$ \\
\hline Mariani 2001 & 0 & 3 & & & & & 0.00 & {$[0.00 ; 0.71]$} & $0.6 \%$ & $0.6 \%$ \\
\hline Danton 1998 & 0 & 13 & & & & & 0.00 & {$[0.00 ; 0.25]$} & $2.5 \%$ & $2.5 \%$ \\
\hline Voets 1997 & 5 & 24 & ए & & & & 0.21 & {$[0.07 ; 0.42]$} & $4.5 \%$ & $4.5 \%$ \\
\hline Rao 1996 & 2 & 30 & 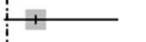 & & & & 0.07 & {$[0.01 ; 0.22]$} & $5.5 \%$ & $5.5 \%$ \\
\hline Francesca 1995 & 1 & 21 & 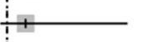 & & & & 0.05 & {$[0.00 ; 0.24]$} & $3.9 \%$ & $3.9 \%$ \\
\hline Brutel 1995 & 5 & 79 & + & & & & 0.06 & {$[0.02 ; 0.14]$} & $14.4 \%$ & $14.4 \%$ \\
\hline Terzi 1994 & 1 & 10 & 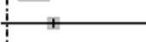 & & & & 0.10 & {$[0.00 ; 0.45]$} & $1.9 \%$ & $1.9 \%$ \\
\hline Miller 1994 & 2 & 30 & & & & & 0.07 & {$[0.01 ; 0.22]$} & $5.5 \%$ & $5.5 \%$ \\
\hline Yokoyama 1993 & 0 & 11 & & & & & 0.00 & {$[0.00 ; 0.28]$} & $2.1 \%$ & $2.1 \%$ \\
\hline Rosalion 1993 & 0 & 10 & & & & & 0.00 & {$[0.00 ; 0.31]$} & $1.9 \%$ & $1.9 \%$ \\
\hline Ulicny 1992 & 1 & 19 & : & & & & 0.05 & {$[0.00 ; 0.26]$} & $3.5 \%$ & $3.5 \%$ \\
\hline Canver 1990 & 1 & 21 & 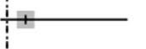 & & & & 0.05 & {$[0.00 ; 0.24]$} & $3.9 \%$ & $3.9 \%$ \\
\hline Fixed effect model & & 536 & 8 & & & & 0.01 & {$[0.00 ; 0.03]$} & $100.0 \%$ & -- \\
\hline Random effects mode & & & $\dot{\Delta}$ & & & & 0.01 & {$[0.00 ; 0.03]$} & ] - & $100.0 \%$ \\
\hline \multicolumn{3}{|c|}{ Heterogeneity: $l^{2}=0 \%, \tau^{2}=0, p=0.79$} & 1 & 1 & 1 & T & & & & \\
\hline & & c & 0.2 & 0.4 & 0.6 & 0.8 & & & & \\
\hline
\end{tabular}




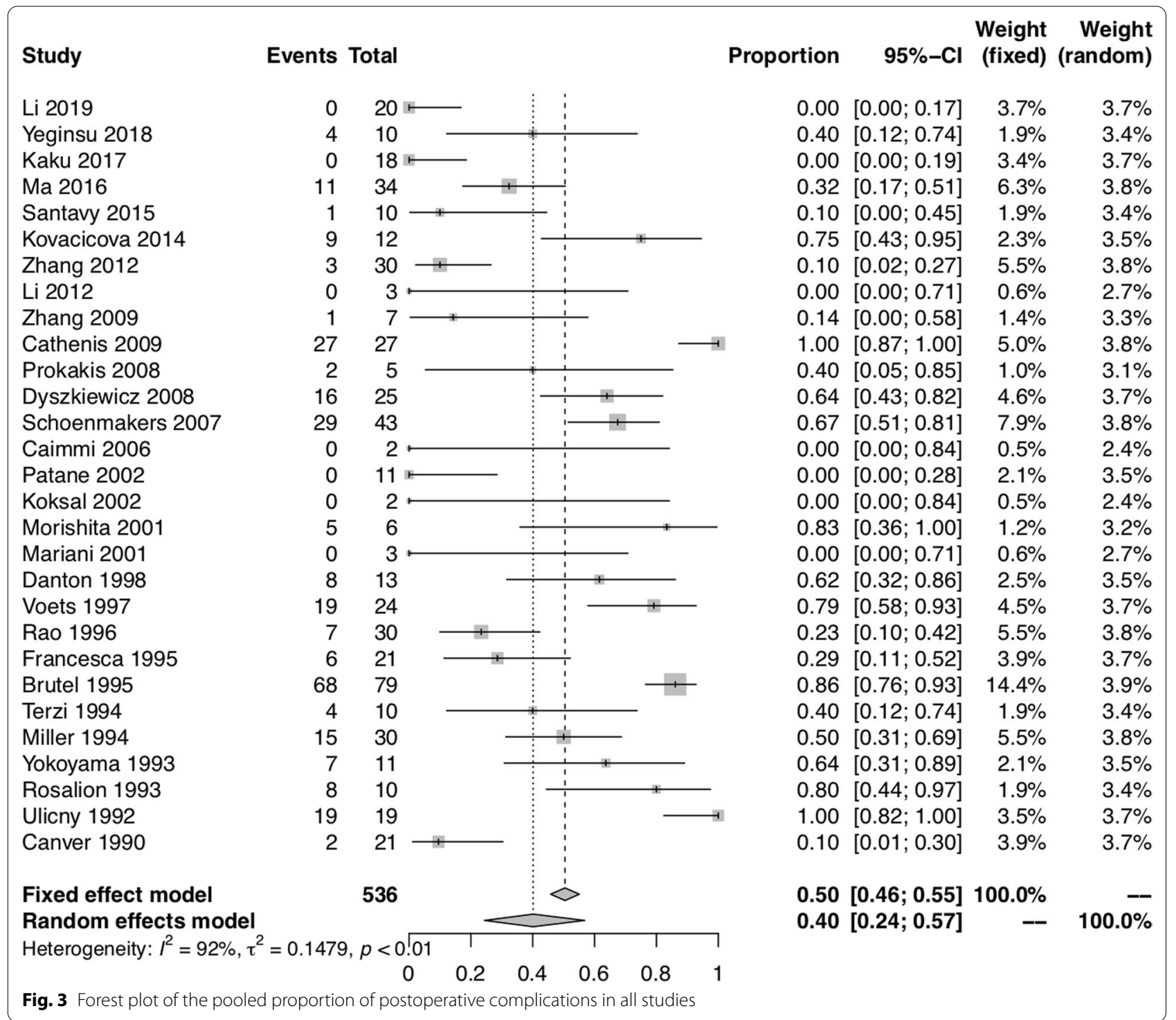

327 patients with lung cancer from the 16 studies [3, $7-9,11,13-16,19,26,28,30,32-34]$. The pooled proportion of operative mortality of patients who received combined heart surgery and lung cancer resection was 0.01 (95\% CI: 0.00, 0.04) using both fixed-effect and random-effect models, with no significant heterogeneity between studies $\left(\mathrm{I}^{2}=2.20 \%, P=0.43\right)$. The pooled proportion of postoperative complications of patients who received combined heart surgery and lung cancer resection was 0.45 (95\% CI: 0.27, 0.64) using the random-effect model, with significant heterogeneity between studies $\left(\mathrm{I}^{2}=87.80 \%, P<0.01\right)$.

To further evaluate the quality control of lung cancer surgery, we investigated whether patients with lung cancer underwent anatomical resection and systematic lymph node dissection or sampling. The pooled proportion of patients with lung cancer who underwent anatomical resection was 0.99 (95\% CI: 0.95, 1.00) using the random-effect model, with significant heterogeneity between studies $\left(\mathrm{I}^{2}=54.70 \%, P<0.01\right)$ (Fig. 5). Among the 16 studies that only included patients with malignant lung lesions [3, 7-9, 11, 13-16, 19, 26, 28, 30, 32-34], 1 study did not provide enough information about lymph node dissection or sampling [7]. Therefore, 15 studies $[3,8,9,11,13-16,19,26,28,30,32-34]$ containing 303 patients who received combined heart surgery and lung cancer resection were used to analyze the proportion of systematic lymph node dissection or sampling. The pooled proportion of patients with lung cancer who underwent systematic lymph node dissection or sampling 


\begin{tabular}{|c|c|c|c|c|c|c|c|c|c|}
\hline Study & Events 7 & Total & & & & Proportion & $95 \%-\mathrm{Cl}$ & $\begin{array}{r}\text { Weight } \\
\text { (fixed) }\end{array}$ & $\begin{array}{r}\text { Weight } \\
\text { (random) }\end{array}$ \\
\hline Li 2019 & 0 & $20 \stackrel{-1+}{3}$ & - & & & 0.00 & {$[0.00 ; 0.17]$} & $3.9 \%$ & $3.9 \%$ \\
\hline Yeginsu 2018 & 1 & 10 & & & & 0.10 & {$[0.00 ; 0.45]$} & $2.0 \%$ & $2.0 \%$ \\
\hline Kaku 2017 & 0 & $18+$ & & & & 0.00 & {$[0.00 ; 0.19]$} & $3.5 \%$ & $3.6 \%$ \\
\hline Ma 2016 & 0 & $34 \stackrel{-}{+}$ & - & & & 0.00 & {$[0.00 ; 0.10]$} & $6.6 \%$ & $6.5 \%$ \\
\hline Santavy 2015 & 0 & $10 \stackrel{4}{4}$ & & & & 0.00 & {$[0.00 ; 0.31]$} & $2.0 \%$ & $2.0 \%$ \\
\hline Kovacicova 2014 & 1 & $12=$ & $一$ & & & 0.08 & {$[0.00 ; 0.38]$} & $2.4 \%$ & $2.4 \%$ \\
\hline Zhang 2012 & 0 & $30 \stackrel{i}{-1}$ & - & & & 0.00 & {$[0.00 ; 0.12]$} & $5.8 \%$ & $5.8 \%$ \\
\hline Li 2012 & 0 & $3+$ & & & & 0.00 & {$[0.00 ; 0.71]$} & $0.7 \%$ & $0.7 \%$ \\
\hline Zhang 2009 & 0 & 7 & & & & 0.00 & {$[0.00 ; 0.41]$} & $1.4 \%$ & $1.5 \%$ \\
\hline Cathenis 2009 & 3 & 27 & 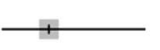 & & & 0.11 & {$[0.02 ; 0.29]$} & $5.2 \%$ & $5.3 \%$ \\
\hline Prokakis 2008 & 0 & $5+$ & & & & 0.00 & {$[0.00 ; 0.52]$} & $1.0 \%$ & $1.1 \%$ \\
\hline Dyszkiewicz 2008 & 1 & 25 & + & & & 0.04 & {$[0.00 ; 0.20]$} & $4.8 \%$ & $4.9 \%$ \\
\hline Schoenmakers 2007 & 0 & $43 \div$ & & & & 0.00 & {$[0.00 ; 0.08]$} & $8.3 \%$ & $8.2 \%$ \\
\hline Caimmi 2006 & 0 & 2 & & & & 0.00 & {$[0.00 ; 0.84]$} & $0.5 \%$ & $0.5 \%$ \\
\hline Patane 2002 & 0 & $11+$ & & & & 0.00 & {$[0.00 ; 0.28]$} & $2.2 \%$ & $2.2 \%$ \\
\hline Koksal 2002 & 0 & $2+$ & & & & 0.00 & {$[0.00 ; 0.84]$} & $0.5 \%$ & $0.5 \%$ \\
\hline Morishita 2001 & 2 & 6 & & & & 0.33 & {$[0.04 ; 0.78]$} & $1.2 \%$ & $1.3 \%$ \\
\hline Mariani 2001 & 0 & $3+$ & & & & 0.00 & {$[0.00 ; 0.71]$} & $0.7 \%$ & $0.7 \%$ \\
\hline Danton 1998 & 0 & 13 & & & & 0.00 & {$[0.00 ; 0.25]$} & $2.6 \%$ & $2.6 \%$ \\
\hline Rao 1996 & 1 & 30 & + & & & 0.03 & {$[0.00 ; 0.17]$} & $5.8 \%$ & $5.8 \%$ \\
\hline Francesca 1995 & 0 & $21 \stackrel{-}{-5}$ & & & & 0.00 & {$[0.00 ; 0.16]$} & $4.1 \%$ & $4.1 \%$ \\
\hline Brutel 1995 & 7 & 79 & + & & & 0.09 & {$[0.04 ; 0.17]$} & $15.1 \%$ & $14.3 \%$ \\
\hline Terzi 1994 & 0 & 10 & & & & 0.00 & {$[0.00 ; 0.31]$} & $2.0 \%$ & $2.0 \%$ \\
\hline Miller 1994 & 2 & 30 & + & & & 0.07 & {$[0.01 ; 0.22]$} & $5.8 \%$ & $5.8 \%$ \\
\hline Yokoyama 1993 & 1 & $11:$ & & & & 0.09 & {$[0.00 ; 0.41]$} & $2.2 \%$ & $2.2 \%$ \\
\hline Rosalion 1993 & 0 & 10 & & & & 0.00 & {$[0.00 ; 0.31]$} & $2.0 \%$ & $2.0 \%$ \\
\hline Ulicny 1992 & 2 & 19 & $\mp$ & & & 0.11 & {$[0.01 ; 0.33]$} & $3.7 \%$ & $3.8 \%$ \\
\hline Canver 1990 & 0 & 21 & & & & 0.00 & {$[0.00 ; 0.16]$} & $4.1 \%$ & $4.1 \%$ \\
\hline Fixed effect model & & $512 \diamond$ & & & & 0.01 & {$[0.00 ; 0.02]$} & $100.0 \%$ & - \\
\hline Random effects mode & & 0 & & & & 0.01 & {$[0.00 ; 0.02]$} & - & $100.0 \%$ \\
\hline \multirow{2}{*}{\multicolumn{3}{|c|}{ Heterogeneity: $I^{2}=2 \%, \tau^{2}=0.0003, p=0.43$}} & $T$ & $T$ & 7 & & & & \\
\hline & & & 0.2 & $0.4 \quad 0.6$ & 0.8 & & & & \\
\hline
\end{tabular}

was 1.00 (95\% CI: 1.00, 1.00) using both fixed-effect and random-effect models, with no significant heterogeneity between studies $\left(\mathrm{I}^{2}=4.20 \%, P=0.41\right)$ (Fig. 6).

In the subgroups classified by heart surgery procedures, 10 studies $[3,9,11,14,15,18,26,28,30,34]$ contained 123 patients who received combined coronary artery bypass grafting (CABG) surgery and lung tumor resection, while the other 19 studies $[5-8,10,12,13,16$, $17,19,20,24,25,27,29,31-33,35]$ enrolled patients who underwent either CABG surgery or other types of heart surgery procedures. Because CABG surgery and other types of heart surgery procedures were analyzed together in these 19 studies $[5-8,10,12,13,16,17,19,20,24,25$, $27,29,31-33,35]$, further assessment of heart surgery procedures other than CABG was not done. Therefore, we quantitatively pooled the data of 123 patients who underwent CABG in 10 studies [3, 9, 11, 14, 15, 18, 26, 28,
$30,34]$. The pooled proportion of operative mortality of patients who received combined CABG surgery and lung tumor resection was 0.00 (95\% CI: $0.00,0.01)$ using both fixed-effect and random-effect models, with no significant heterogeneity between studies $\left(\mathrm{I}^{2}=0.00 \%, P=0.76\right)$. The pooled proportion of postoperative complications of patients who received combined CABG surgery and lung tumor resection was 0.26 (95\% CI: $0.07,0.50)$ using the random-effect model, with significant heterogeneity between studies $\left(\mathrm{I}^{2}=79.80 \%, P<0.01\right)$.

To further evaluate the impact of $\mathrm{CPB}$ on postoperative complications, we compared the effect of the off-pump versus the on-pump method on postoperative complications in patients who underwent CABG. The pooled proportion of postoperative complications of patients who underwent CABG using the off-pump method was 0.17 (95\% CI: 0.01, 0.43) using the random-effect model, with 


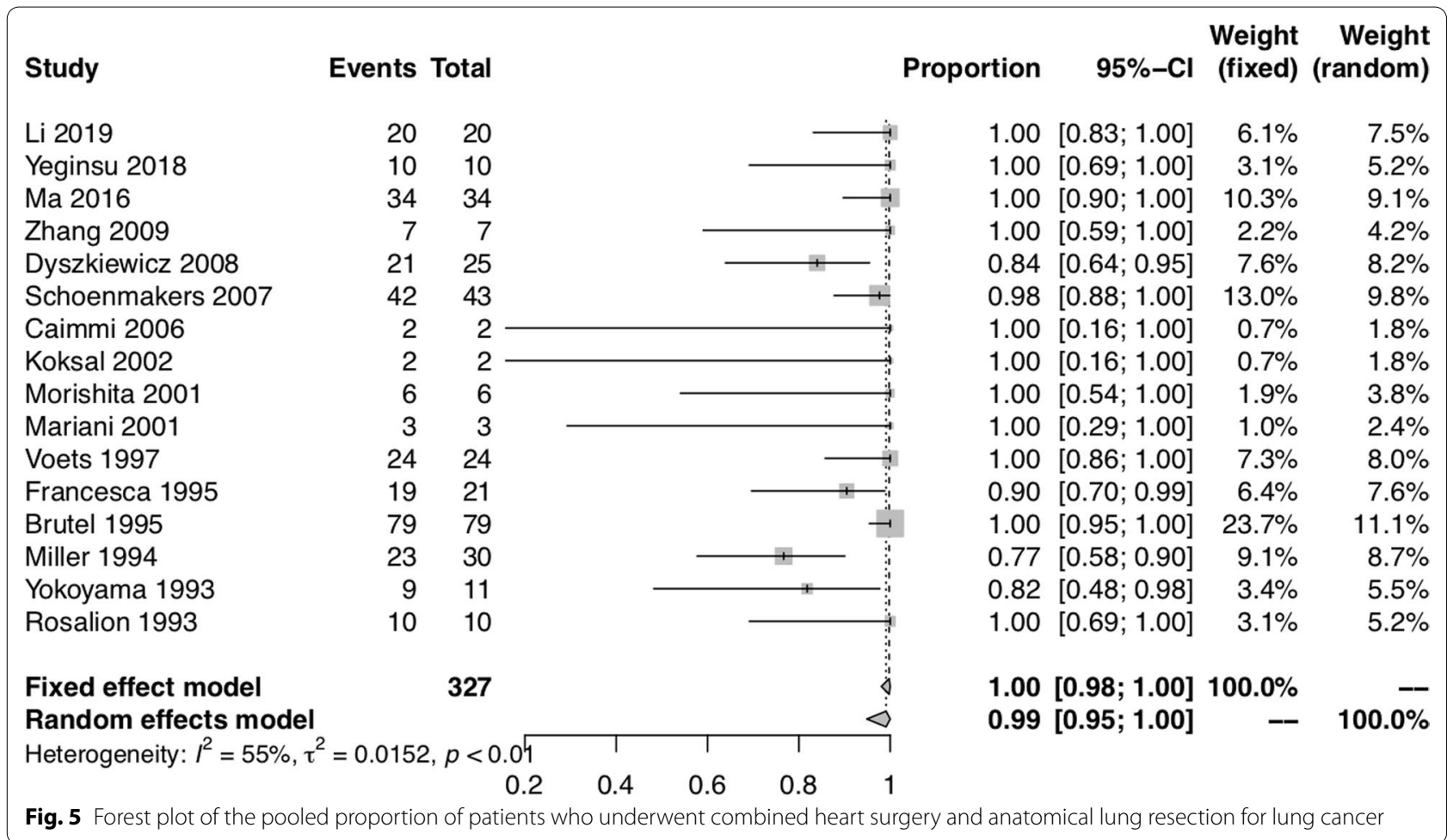

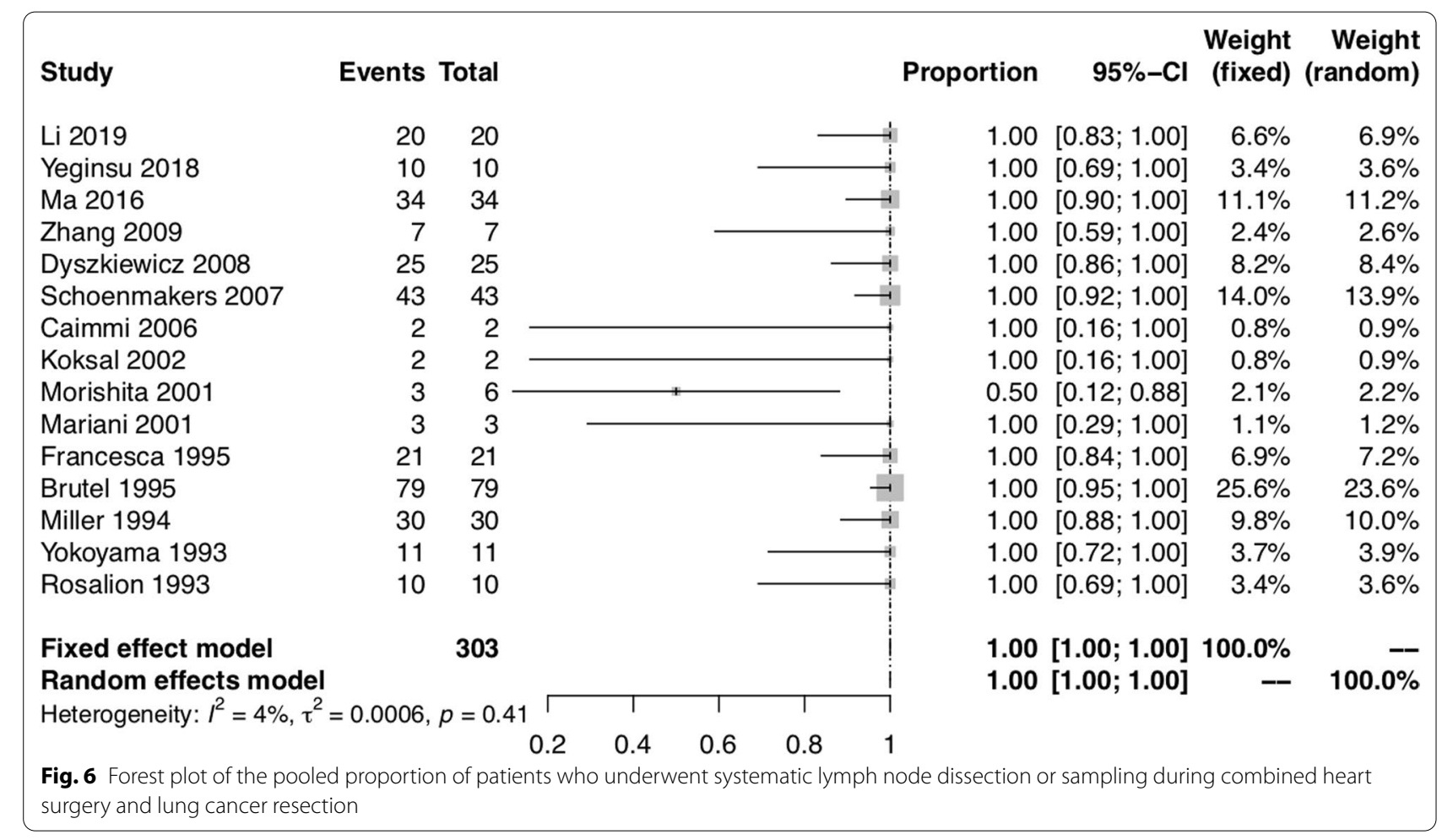


significant heterogeneity between studies $\left(\mathrm{I}^{2}=79.40 \%\right.$, $P<0.01)$. The pooled proportion of postoperative complications of patients who underwent CABG using the on-pump method was 0.61 (95\% CI: 0.38, 0.82) using the fixed-effect model, with no significant heterogeneity between studies $\left(\mathrm{I}^{2}=67.40 \%, P=0.08\right)$ (Fig. 7).

\section{Sensitivity analysis and publication bias}

Sensitivity analysis showed that no individual study influenced the overall results (Fig. 8). Funnel plot for publication bias of the pooled proportion of postoperative complications for all studies was shown in Fig. 9. There was no significant publication bias detected using Begg's test $(P=0.42)$ and Egger's test $(P=0.07)$.

\section{Discussion}

This systematic review and meta-analysis involved a total of 536 patients who received combined heart surgery and lung tumor resection in 29 observational studies. Our comprehensive data analysis demonstrated that combined heart surgery and lung tumor resection had a low mortality rate and an acceptable complication rate. The incidence rate of reopening for bleeding was quite low. The incidence rates of postoperative respiratory complications and cardiac complications were similar. Overall, the present results suggest that combined heart surgery and lung tumor resection is safe with low mortality and complication rates.

A previous systematic review by Tourmousoglou and associates included 15 retrospective studies from 1994 to 2012 [36]. They compared the operative mortality rate, reoperation rate, and survival rate between combined and staged surgery for patients with both coronary artery disease and lung cancer. Without quantitative comparison, the authors found that the operative mortality rate was $0-20.8 \%$ for combined procedures and $0-10 \%$ for staged procedures. The reoperation rate for bleeding was $0-11 \%$ for combined procedures and $0 \%$ for staged procedures. Similar to our results, a more recent systematic review and meta-analysis by Bablekos and co-workers included 22 retrospective studies from 1985 to 2011 [37]. They summarized the operative mortality rate, complication rate, and survival rate of lung cancer patients complicated with heart disease. Without performing subgroup analysis of simultaneous or staged surgery, they found the pooled operative mortality rate was $5.26 \%$ (95\% CI: $3.47,7.62)$ and the pooled complication rate was $45.59 \%$ (95\% CI: 35.62, 55.74).

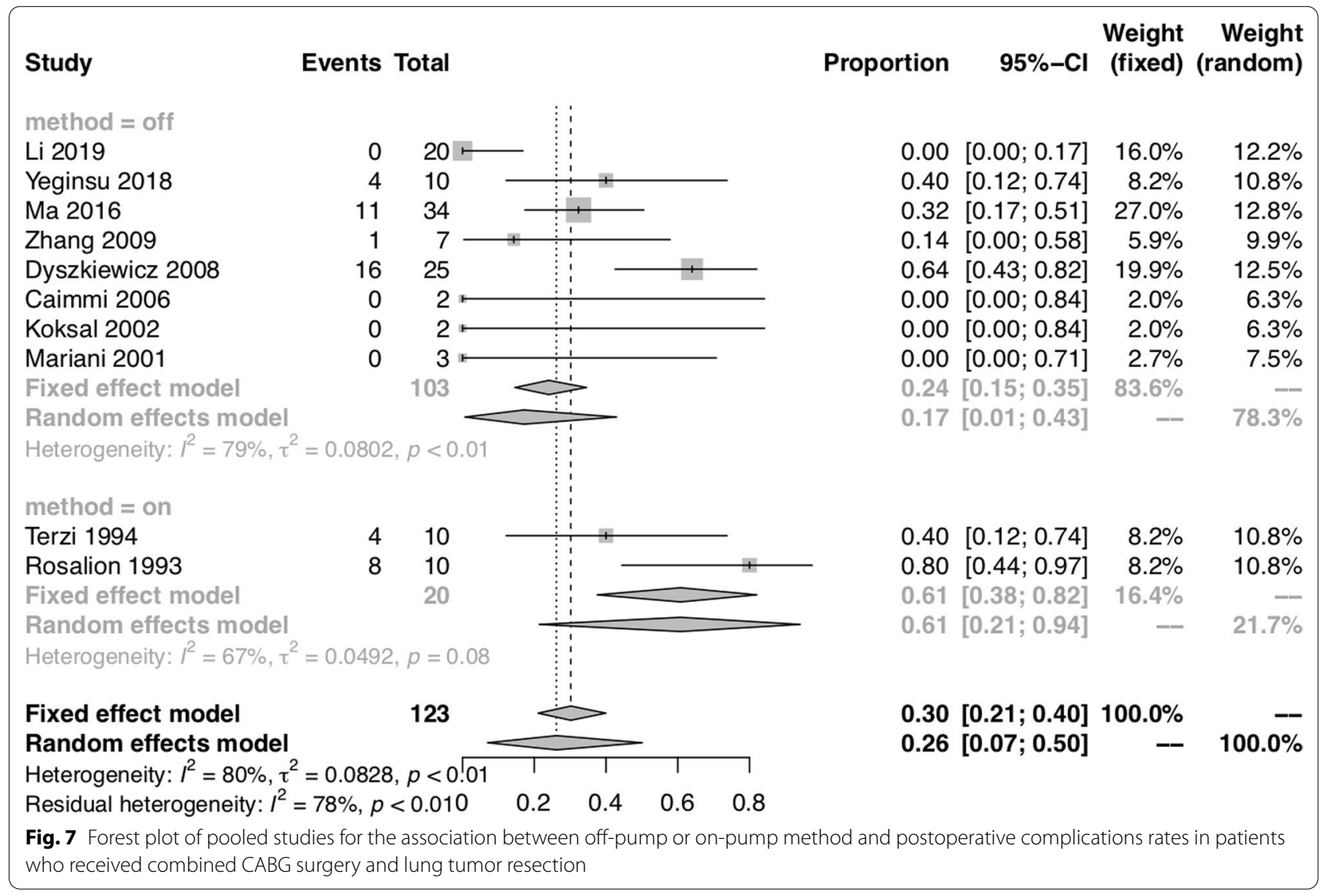




\section{Study}

Omitting Li 2019

Omitting Yeginsu 2018

Omitting Kaku 2017

Omitting Ma 2016

Omitting Santavy 2015

Omitting Kovacicova 2014

Omitting Zhang 2012

Omitting Li 2012

Omitting Zhang 2009

Omitting Cathenis 2009

Omitting Prokakis 2008

Omitting Dyszkiewicz 2008

Omitting Schoenmakers 2007

Omitting Caimmi 2006

Omitting Patane 2002

Omitting Koksal 2002

Omitting Morishita 2001

Omitting Mariani 2001

Omitting Danton 1998

Omitting Voets 1997

Omitting Rao 1996

Omitting Francesca 1995

Omitting Brutel 1995

Omitting Terzi 1994

Omitting Miller 1994

Omitting Yokoyama 1993

Omitting Rosalion 1993

Omitting Ulicny 1992

Omitting Canver 1990

Random effects model

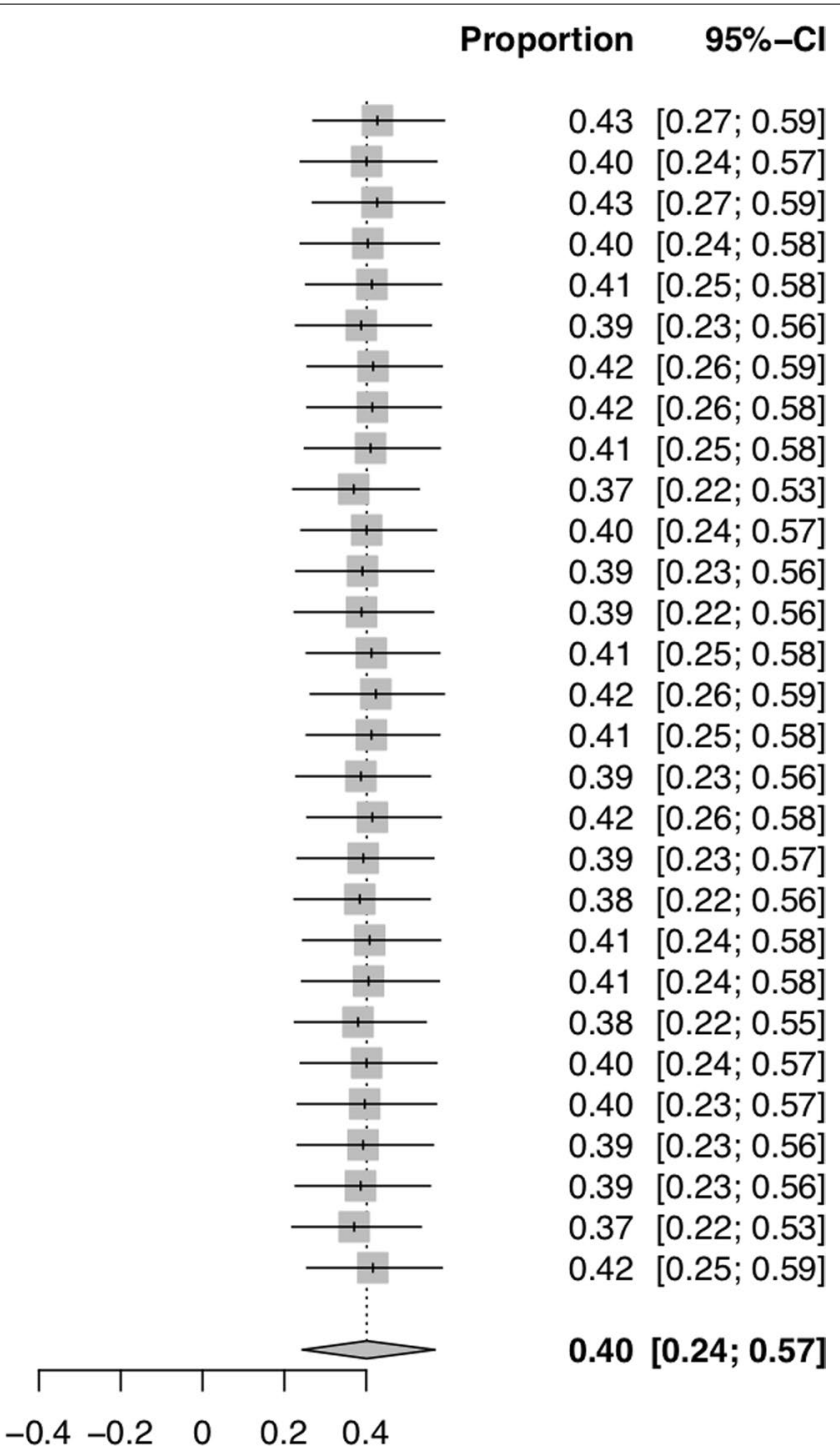

$\begin{array}{lllll}-0.4 & -0.2 & 0 & 0.2 & 0.4\end{array}$
$0.43[0.27 ; 0.59]$

$0.40[0.24 ; 0.57]$

$0.40[0.27 ; 0.59]$

$0.41[0.25 ; 0.58]$

$0.39[0.23 ; 0.56]$

$0.42[0.26 ; 0.59]$

$0.42[0.26 ; 0.58]$

$0.41[0.25 ; 0.58]$

$0.37[0.22 ; 0.53]$

$0.40[0.24 ; 0.57]$

$0.39[0.23 ; 0.56]$

$0.39[0.22 ; 0.56]$

$0.41[0.25 ; 0.58]$

$0.42[0.26 ; 0.59]$

$0.41[0.25 ; 0.58]$

$0.39[0.23 ; 0.56]$

$0.42[0.26 ; 0.58]$

$0.39[0.23 ; 0.57]$

$0.38[0.22 ; 0.56]$

$0.41[0.24 ; 0.58]$

$0.41[0.24 ; 0.58]$

$0.38[0.22 ; 0.55]$

$0.40[0.24 ; 0.57]$

$0.40[0.23 ; 0.57]$

$0.39[0.23 ; 0.56]$

$0.39[0.23 ; 0.56]$

$0.37[0.22 ; 0.53]$

$0.42[0.25 ; 0.59]$

$0.40[0.24 ; 0.57]$

Fig. 8 Sensitivity analysis for the pooled proportion of postoperative complications for all studies

Patients who undergo combined heart surgery and lung tumor resection face a potential risk of postoperative bleeding. This concern raises from $\mathrm{CPB}$ use during heart surgery and, while performing heart and lung surgery simultaneously, the more complicated surgical procedure. Potential bleeding might be related to excessive heparin use, inadequate heparin neutralization, or platelet dysfunction during CPB [38, 39]. Bleeding might arise from the area of the lung resection, extensive mediastinal lymph node dissection and, not less frequently, intrapulmonary hemorrhage [20, 40]. Excessive bleeding increases the reexploration rate and eventually increases the mortality and complication rates. Off-pump CABG is one solution to reduce the potential risk of postoperative bleeding. During off-pump CABG, the intraoperative heparin dose was reduced and the platelet functions were less affected, leading to decreased bleeding [41]. Alternatively, when $\mathrm{CPB}$ was necessary, the lung resection and lymph node dissection were best performed before heparinization or after reversal of heparin by protamine sulfate $[19,35]$. Furthermore, compared with wedge excisions, anatomical lung resection was preferred to reduce the risk of intrapulmonary hemorrhage [20,40].

In the subgroups classified by lung pathology, we focused on patients who underwent combined heart surgery and lung cancer resection. As median sternotomy was often employed as a single incision to perform simultaneous cardiac and pulmonary surgery, there were 


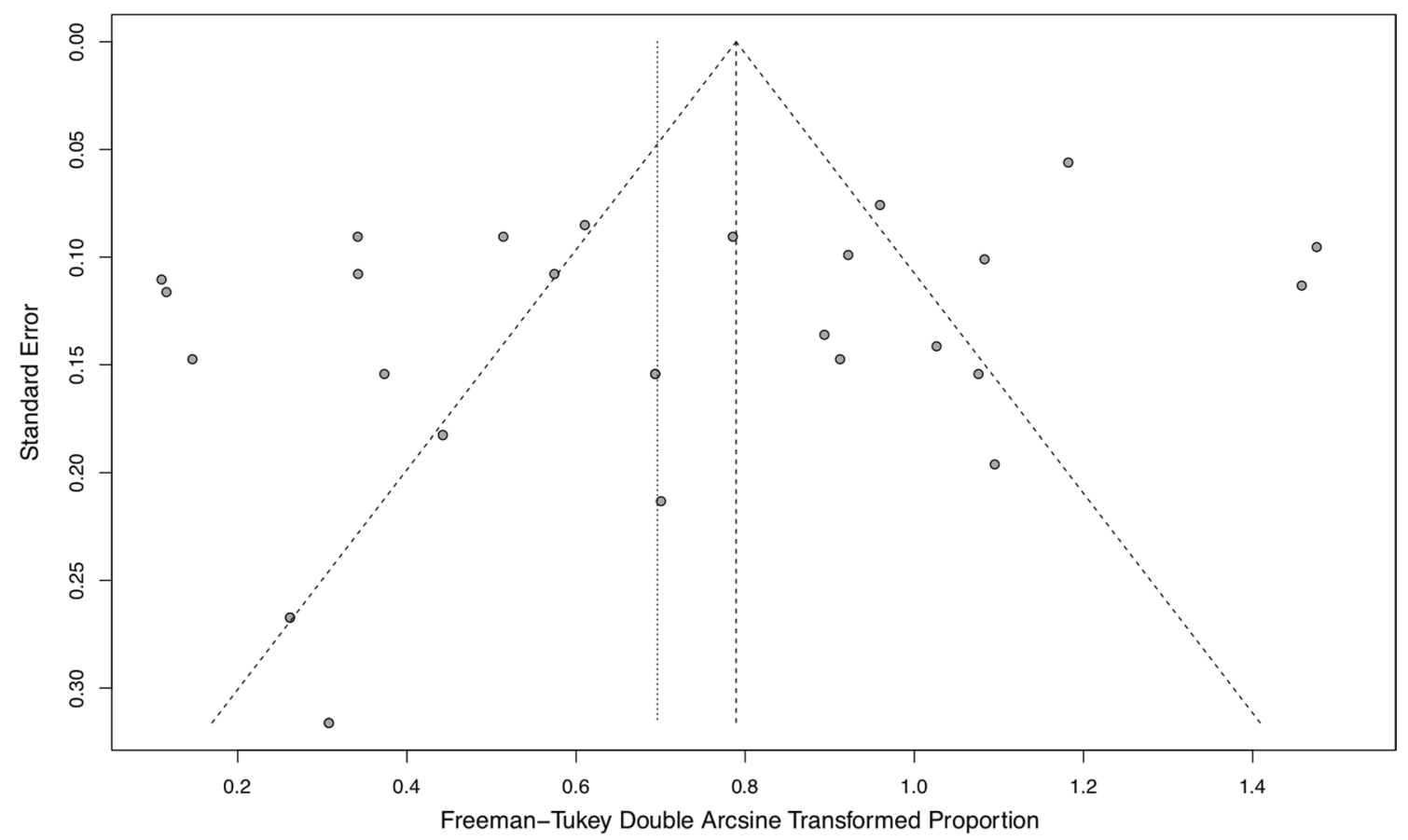

Fig. 9 Funnel plot for publication bias of the pooled proportion of postoperative complications for all studies

concerns regarding the safety and adequacy of oncological pulmonary and lymph node resection [6, 8, 14, 17]. Through a median sternotomy, left lower lobectomy was considered the most technically difficult pulmonary lobectomy $[9,12,16,33,42]$. The intraoperative view of left lower lobectomy was obscured by the heart. Retraction of the heart was often needed, but possibly led to arrhythmias and hemodynamic instability. To facilitate exposure of the left lower lobe, $\mathrm{CPB}$ could be used to reduce the heart volume. Also, adding a lateral thoracotomy to the median sternotomy or extending the median sternotomy into the intercostal space could be adopted. Compared with a lateral thoracotomy, performing mediastinal lymph node dissection through a median sternotomy was more technically difficult and time-consuming. Although systematic mediastinal lymph node dissection through a median sternotomy was possible, the subcarinal and posterior mediastinal lymph nodes were particularly difficult to sample $[8,31]$. One solution was to open the posterior pericardium and mobilize the right pulmonary artery, allowing a complete dissection of the subcarinal space. Also, adding a lateral thoracotomy to the median sternotomy could be adopted.

In the subgroups classified by heart surgery procedures, we focused on patients who underwent combined CABG surgery and lung tumor resection. We compared the effect of off-pump versus on-pump CABG on postoperative complications and found that off-pump CABG could reduce the complication rate. Many disadvantages of CPB have been described. With higher doses of heparin, CPB may increase the risk of bleeding [39]. In addition, $\mathrm{CPB}$ circuits manufactured from synthetic materials may impair platelet function and enhance systemic inflammatory response syndrome [43]. Furthermore, CPB may cause edema of the peribronchial and lung tissue, as well as lung injury [44-46], and may impair the immune system and promote tumor cell metastasis [47]. After eliminating the shortcomings of $\mathrm{CPB}$, off-pump CABG might reduce the complication rate.

The present meta-analysis has several limitations that should be acknowledged. First, there was significant heterogeneity between included studies, which was likely due to clinical and methodological differences between studies. Second, all included studies were retrospective observational studies. Third, survival data were not evaluated in our study. Our study focused on perioperative outcomes such as the incidence rates of operative mortality and postoperative complications. Fourth, articles comparing combined and staged surgery were so limited. Only three articles were found from our comprehensive literature search. So, the comparison of mortality and complication rates of combined cardiothoracic surgery and staged surgery was not performed in our study. More studies are needed to compare the mortality and morbidity of combined and staged surgery. Fifth, none of the included studies 
had described in detail whether complete or incomplete revascularization was achieved in coronary artery disease patients. So, the comparison between complete and incomplete revascularization for coronary artery disease patients was not performed in our study.

\section{Conclusion}

Our systematic meta-analysis demonstrated that combined heart surgery and lung tumor resection had a low mortality rate and an acceptable complication rate. According to the subgroup analysis, although the procedures were technically difficult and time-consuming, most patients with lung cancer successfully underwent anatomical resection and mediastinal lymph node sampling or dissection. For patients receiving combined CABG surgery and lung tumor resection, off-pump CABG may potentially reduce the complication rate compared with on-pump CABG. Considering the limitations of this study, our findings need to be further confirmed in large-scale prospective research.

\section{Abbreviations \\ CABG: Coronary artery bypass grafting; CPB: Cardiopulmonary bypass; NOS: Newcastle-Ottawa Scale.}

\section{Acknowledgements}

We thank Kelly Zammit, BVSc, from Liwen Bianji (Edanz) (www.liwenbianji.cn/), for editing the English text of a draft of this manuscript.

\section{Authors' contributions}

SC and YJ collected the data and drafted the manuscript. SC, XL, XL, XZ and DS edited the manuscript, participated in the study design and coordination, and helped to draft the manuscript. All authors read and approved the final manuscript.

\section{Funding}

This work was supported by grants from the National Natural Science Foundation of China (81800214), Natural Science Foundation of Anhui Province (1808085QH236).

\section{Availability of data and materials}

All data generated or analyzed during this study are included in this published article.

\section{Declarations}

Ethics approval and consent to participate

Not applicable.

\section{Consent for publication}

Not applicable.

\section{Competing interests}

The authors declare that they have no competing interests.

\section{Author details}

'Department of Thoracic Surgery, Tianjin Chest Hospital, Tianjin 300222, China. ${ }^{2}$ Department of Cardiac Surgery, Tianjin First Central Hospital, Tianjin 300074, China.
Received: 28 September 2020 Accepted: 2 August 2021

Published online: 09 August 2021

\section{References}

1. Barta JA, Powell CA, Wisnivesky JP. Global epidemiology of lung cancer. Ann Glob Health. 2019;85(1):8.

2. Khan MA, Hashim MJ, Mustafa H, Baniyas MY, Al Suwaidi SKBM, AlKatheeri $R$, et al. Global epidemiology of ischemic heart disease: results from the global burden of disease study. Cureus. 2020;12(7):e9349.

3. Li Z, Liu B, Ge W, Zhang W, Gu C, Liu J, et al. Effect of simultaneous surgical treatment of severe coronary artery disease and lung cancer. J Int Med Res. 2019:47(2):591-9.

4. Ciriaco P, Carretta A, Calori G, Mazzone P, Zannini P. Lung resection for cancer in patients with coronary arterial disease: analysis of short-term results. Eur J Cardiothorac Surg. 2002;22(1):35-40.

5. Zhang R, Wiegmann B, Fischer S, Dickgreber NJ, Hagl C, Kruger M, et al. Simultaneous cardiac and lung surgery for incidental solitary pulmonary nodule: learning from the past. Thorac Cardiovasc Surg. 2012;60(2):150-5.

6. Cathenis K, Hamerlijnck R, Vermassen F, Van Nooten G, Muysoms F. Concomitant cardiac surgery and pulmonary resection. Acta Chir Belg. 2009;109(3):306-11.

7. Voets AJ, Joesoef KS, van Teeffelen ME. Synchroneously occurring lung cancer (stages I-II) and coronary artery disease: concomitant versus staged surgical approach. Eur J Cardiothorac Surg. 1997;12(5):713-7.

8. Miller DL, Orszulak TA, Pairolero PC, Trastek VF, Schaff HV. Combined operation for lung cancer and cardiac disease. Ann Thorac Surg. 1994:58(4):989-93 (discussion 93-4).

9. Yeginsu A, Vayvada M, Karademir BC, Erkilinc A, Tasci AE, Buyukbayrak $F$, et al. Combined off-pump coronary artery bypass grafting and lung resection in patients with lung cancer accompanied by coronary artery disease. Braz J Cardiovasc Surg. 2018;33(5):483-9.

10. Kaku R, Teramoto K, Ishida K, Igarashi T, Hashimoto M, Kitamura S, et al. Simultaneous resection of pulmonary tumor following cardiovascular surgery. Asian J. 2017;40(2):123-8.

11. Ma X, Huang F, Zhang Z, Song F, Ou S. Lung cancer resection with concurrent off-pump coronary artery bypasses: safety and efficiency. J Thorac Dis. 2016;8(8):2038-45.

12. Santavy P, Szkorupa M, Bohanes T, Lonsky V. Simultaneous cardiac surgery with pulmonary resection. Cor Vasa. 2015;57(2):e82-5.

13. Schoenmakers MC, van Boven WJ, van den Bosch J, van Swieten HA. Comparison of on-pump or off-pump coronary artery revascularization with lung resection. Ann Thorac Surg. 2007;84(2):504-9.

14. Caimmi PP, Di Biasi P. Combined minimally invasive coronary bypass surgery and left pulmonary lobectomy. Asian Cardiovasc Thorac Ann 2006:14(3):250-1.

15. Koksal C, Sarikaya S, Zengin M, Atasalihi A. Combined off-pump coronary revascularization and lung resection. Acta Medica (Hradec Kralove). 2002;45(3):119-21.

16. Morishita K, Kawaharada N, Watanabe T, Koshima R, Fujisawa Y, Watanabe A, et al. Simultaneous cardiac operations with pulmonary resection for lung carcinoma. Jpn J Thorac Cardiovasc Surg. 2001;49(12):685-9.

17. Danton MH, Anikin VA, McManus KG, McGuigan JA, Campalani G. Simultaneous cardiac surgery with pulmonary resection: presentation of series and review of literature. Eur J Cardiothorac Surg. 1998;13(6):667-72.

18. Terzi A, Furlan G, Magnanelli G, Conti F, Chiavacci P, Petrilli G, et al. Lung resections concomitant to coronary artery bypass grafting. Eur J Cardiothorac Surg. 1994;8(11):580-4.

19. Yokoyama T, Derrick MJ, Lee AW. Cardiac operation with associated pulmonary resection. J Thorac Cardiovasc Surg. 1993;105(5):912-6 (discussion 6-7).

20. Ulicny KS Jr, Schmelzer V, Flege JB Jr, Todd JC, Mitts DL, Melvin DB, et al. Concomitant cardiac and pulmonary operation: the role of cardiopulmonary bypass. Ann Thorac Surg. 1992;54(2):289-95.

21. Barendregt JJ, Doi SA, Lee YY, Norman RE, Vos T. Meta-analysis of prevalence. J Epidemiol Community Health. 2013;67(11):974-8.

22. Higgins JP, Thompson SG. Quantifying heterogeneity in a meta-analysis. Stat Med. 2002;21(11):1539-58. 
23. Egger M, Smith GD, Schneider M, Minder C. Bias in meta-analysis detected by a simple, graphical test. BMJ. 1997;315(7109):629-34.

24. Kovacicova K, Omran N, Mandak J. Combined surgical treatment of lung cancer and heart diseases. Bratisl Lek Listy. 2014;115(12):776-80.

25. Li W, Lei W, Li B, Chen X, Ma L, Ni Y. Experience of simultaneous coronary artery bypass grafting and lung lobectomy. Chirurgia. 2012;25(3):173-7.

26. Zhang $Y$, Wei $X$, Pan T. Simultaneous surgical treatment of non-small cel lung cancer and off-pump coronary artery bypass grafting. Chin J Lung Cancer. 2009:12(4):332-6.

27. Prokakis C, Koletsis E, Apostolakis E, Panagopoulos N, Charoulis N, Velissaris $D$, et al. Combined heart surgery and lung tumor resection. Med Sci Monit. 2008;14(3):CS17-21.

28. Dyszkiewicz W, Jemielity M, Piwkowski C, Kasprzyk M, Perek B, Gasiorowski L, et al. The early and late results of combined off-pump coronary artery bypass grafting and pulmonary resection in patients with concomitant lung cancer and unstable coronary heart disease. Eur J Cardiothorac Surg. 2008;34(3):531-5.

29. Patane F, Verzini A, Zingarelli E, di Summa M. Simultaneous operation for cardiac disease and lung cancer. Interact Cardiovasc Thorac Surg. 2002;1(2):69-71.

30. Mariani MA, van Boven WJ, Duurkens VA, Ernst SM, van Swieten HA. Combined off-pump coronary surgery and right lung resections through midline sternotomy. Ann Thorac Surg. 2001;71(4):1343-4.

31. Rao V, Todd TR, Weisel RD, Komeda M, Cohen G, Ikonomidis JS, et al. Results of combined pulmonary resection and cardiac operation. Ann Thorac Surg. 1996;62(2):342-6 (discussion 6-7).

32. La Francesca S, Frazier OH, Radovancevic B, De Caro LF, Reul GJ, Cooley DA. Concomitant cardiac and pulmonary operations for lung cancer. Tex Heart Inst J. 1995;22(4):296-300.

33. Brutel de la Riviere A, Knaepen P, Van Swieten $H$, Vanderschueren R, Ernst J, Van den Bosch J. Concomitant open heart surgery and pulmonary resection for lung cancer. Eur J Cardiothorac Surg. 1995;9(6):310-3 (discussion 3-4).

34. Rosalion A, Woodford NW, Clarke CP, Buxton B. Concomitant coronary revascularization and resection of lung cancer. Aust N Z J Surg. 1993;63(5):336-40.

35. Canver CC, Bhayana JN, Lajos TZ, Raza ST, Lewin AN, Bergsland J, et al. Pulmonary resection combined with cardiac operations. Ann Thorac Surg. 1990;50(5):796-9.

36. Tourmousoglou CE, Apostolakis E, Dougenis D. Simultaneous occurrence of coronary artery disease and lung cancer: what is the best surgical treatment strategy? Interact Cardiovasc Thorac Surg. 2014;19(4):673-81.
37. Bablekos GD, Analitis A, Michaelides SA, Charalabopoulos KA, Tzonou A. Management and postoperative outcome in primary lung cancer and heart disease co-morbidity: a systematic review and meta-analysis. Ann Transl Med. 2016;4(11):213.

38. Ranucci M, Pistuddi V, Di Dedda U, Menicanti L, De Vincentiis C, Baryshnikova E. Platelet function after cardiac surgery and its association with severe postoperative bleeding: the PLATFORM study. Platelets. 2019;30(7):908-14.

39. Kunz SA, Miles LF, lanno DJ, Mirowska-Allen KL, Matalanis G, Bellomo $R$, et al. The effect of protamine dosing variation on bleeding and transfusion after heparinisation for cardiopulmonary bypass. Perfusion. 2018;33(6):445-52.

40. Piehler JM, Trastek VF, Pairolero PC, Pluth JR, Danielson GK, Schaff HV, et al. Concomitant cardiac and pulmonary operations. J Thorac Cardiovasc Surg. 1985;90(5):662-7.

41. Gaudino M, Angelini GD, Antoniades C, Bakaeen F, Benedetto U, Calafiore AM, et al. Off-pump coronary artery bypass grafting: 30 years of debate. J Am Heart Assoc. 2018;7(16):e009934.

42. Liu B, Chen C, Gu C, Li Q, Liu J, Pu Y, et al. Combined coronary artery bypass graft ( $C A B G)$ surgery and lung resection for lung cancer in patients more than 50 years-of-age. Med Sci Monit. 2018;24:3307.

43. Bronicki RA, Hall M. Cardiopulmonary bypass-induced inflammatory response: pathophysiology and treatment. Pediatr Crit Care Med. 2016:17(8):S272-8.

44. Hou L, Yang Z, Wang Z, Zhang X, Zhao Y, Yang H, et al. NLRP3/ASCmediated alveolar macrophage pyroptosis enhances HMGB1 secretion in acute lung injury induced by cardiopulmonary bypass. Lab Invest. 2018;98(8):1052-64.

45. Apostolakis E, Filos KS, Koletsis E, Dougenis D. Lung dysfunction following cardiopulmonary bypass. J Card Surg. 2010;25(1):47-55.

46. Wynne R, Botti M. Postoperative pulmonary dysfunction in adults after cardiac surgery with cardiopulmonary bypass: clinical significance and implications for practice. Am J Crit Care. 2004;13(5):384-93.

47. Hübner M, Tomasi R, Effinger D, Wu T, Klein G, Bender M, et al. Myeloidderived suppressor cells mediate immunosuppression after cardiopulmonary bypass. Crit Care Med. 2019;47(8):e700-9.

\section{Publisher's Note}

Springer Nature remains neutral with regard to jurisdictional claims in published maps and institutional affiliations.
Ready to submit your research? Choose BMC and benefit from:

- fast, convenient online submission

- thorough peer review by experienced researchers in your field

- rapid publication on acceptance

- support for research data, including large and complex data types

- gold Open Access which fosters wider collaboration and increased citations

- maximum visibility for your research: over 100M website views per year

At BMC, research is always in progress.

Learn more biomedcentral.com/submissions 